\title{
Simulation of Radial Variation of Neutral Atoms on Edge Plasma of Small Size Divertor Tokamak
}

\author{
Amr Hasheim Bekheit \\ Plasma and Nuclear Fusion Department, Atomic Energy Authority, Cairo, Egypt \\ Email: amrbekheitga@yahoo.com
}

Received August 19, 2011; revised October 26, 2011; accepted November 18, 2011

\begin{abstract}
Simulations have been performed to investigate the impact of radial variation of neutral atoms (neutral puff) on the edge plasma of small size divertor tokamak. It was demonstrated that, the variation of neutral atoms (neutral puff) in edge plasma of small size divertor tokamak generates additional large radial electric field and large radial electric field shear near separatrix which can significantly influence global confinement by affecting the transition from low (L) to high (H) confinement. This simulation was performed by using B2SOLPS0.5.2D fluid transport code based on a reduced form of the transport form of transport equations. These transport equations are implemented in B2SOLPS0.5.2D fluid transport code and solved for the parameters of Small Size Divertor tokamak. The results of simulation by fluid transport B2SOLPS0.5.2D code can be summarized as follow: 1) The Plasma Parameters are significantly effect by neutral atoms puffing in the edge plasma of small size divertor tokamak; 2) Contrary to previous expectation [1] of the predominant role of neutral viscosity on toroidal flux, anomalous transport was found to be mainly effect on toroidal flux; 3) Puffing of gas (neutral) in the edge plasma of small size divertor tokamak produce strong ITB; 4) Puffing of gas (neutral) in the edge plasma of small size divertor increase plasma density especially in SOL through charge exchange and ionization processes; 5) Puffing of gas (neutral) in the edge plasma of small size divertor has significant effect on the distribution of plasma heat flux; 6) The radial electric field is affected by gas (neutral) puffing in the edge plasma of small size divertor; 7) Puffing of neutral (atoms) in the edge plasma of small size divertor tokamak produce large radial electric field shear which contribute to L-H transition; 8) The centrifugal effect has no influence on distribution of the radial profile of parallel (toroidal) velocity of edge plasma of small size divertor tokamak during gas (neutral) puffing; 9) The bootstrap current in edge plasma of small size divertor tokamak is significantly affected by gas (neutral) puffing.
\end{abstract}

Keywords: Neutral Puffing; Transport Code; Radial Electric Field

\section{Introduction}

Neutral atoms in tokamak edge plasma can significantly influence global confinement by affecting the transition from low $(\mathrm{L})$ to high $(\mathrm{H})$ confinement The neutrals affect the ion dynamics through charge exchange (CX), and the neutral flux of toroidal angular momentum can modify or even determine the edge radial electric field and the plasma rotation [1]. The localization of the neutrals introduces a shear in the flow that may also affect edge turbulence. Previous results suggest an external means for controlling toroidal flow and radial electric field at the edge that is being investigated on MAST [2,3]. In recent experiments on MAST [2,3] it was observed that toroidal rotation inside the separatrix depends on the position of gas (neutral) puff [2]. For MAST tokamak the inboard gas (neutral) puff the rotation at the low field side (LFS) was counter-current directed while for outboard puff the direction of toroidal velocity was co-current. Co-current toroidal rotation at the outer midplane for ion $\nabla \mathrm{B}$ drift directed towards the X-point has been observed in the scrape off layer (SOL) on several tokomaks [3,4]. The simulation with B2SOLPS [5-7], UEDGE [6] and EDGE2D [8] reproduced rotation in the same direction. However, recent work on small size divertor tokamak indicates that the poloidal distribution of neutral has essential influence on L-H transition threshold power. Moreover, gas puff increased the plasma temperature, plasma density and radial electric field in the edge plasma of this tokamak. Therefore, in the present paper modeling of the impact of gas (neutral) puff on tokamak edge plasma is performed by means of the B2SOLPS0.5.2D fluid transport code for NBI shots for small size divertor tokamak. The impact of gas (neutral) puff on plasma parameters, plasma heat fluxes and radial electric field are investigated.

\section{The Main Simulation Results}

The simulation was performed for L-regime of small size divertor tokamak (SSDT) the global parameters of dis- 
charge are listed in Table 1. The direction of $\boldsymbol{\nabla} \boldsymbol{B}$-drift in the discharge was upwards. The temperature of ion $T_{i}=1$ keV and plasma density $n_{\mathrm{i}}=n_{\mathrm{e}}=n=4 \times 10^{19} \mathrm{~m}^{-3}$.

The anomalous values of the diffusion and heat conductivity coefficients were chosen equal for all sort of particle $D=0.5 \mathrm{~m}^{2} \cdot \mathrm{s}^{-1}, \chi_{\mathrm{e}, \mathrm{i}}=0.7 \mathrm{~m}^{2} \cdot \mathrm{s}^{-1}$. The simulations were performed with B2SOLPS0.5.2D fluid transport code. As in similar codes the set of modified Braginski equations was solved $[5,6]$. The philosophy B2SOLPS0.5.2D fluid transport code (and other codes) is that the values of perpendicular transport coefficients are chosen to fit experimentally observed density, temperature radial

Table 1. Global plasma parameter for SSDT.

\begin{tabular}{cc}
\hline Plasma current & $I=50 \mathrm{kA}$ (counter-clockwise) \\
\hline Toroidal magnetic field & $B_{T}=1.7 \mathrm{~T}$ \\
Major and minor radii & $R=0.3 \mathrm{~m}, a=0.1 \mathrm{~m}$ \\
\hline
\end{tabular}

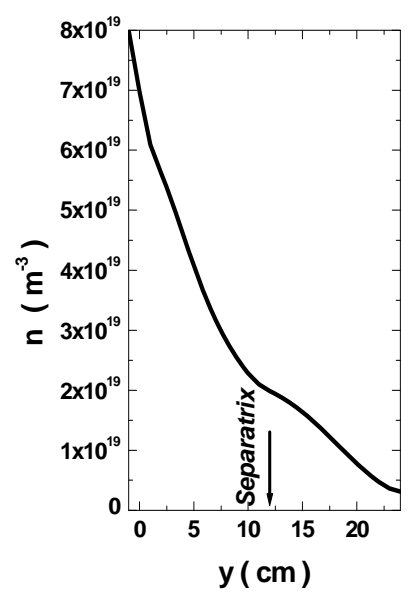

Figure 1. Plasma density distribution in edge plasma of SSDT without gas puff.

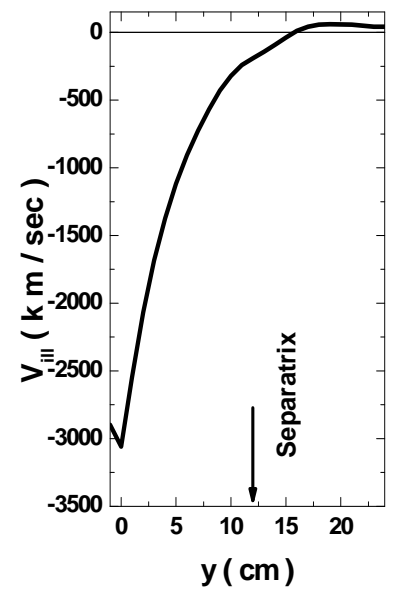

Figure 3. Parallel velocity distribution in edge plasma of SSDT without gas puff. profiles, density and temperature near the divertor plates. In the simulation presented below the perpendicular transport coefficients are replaced by the anomalous values: diffusion, electron, ion heat flux and perpendicular viscosity coefficients [5]. The perpendicular (anomalous) viscosity coefficient was taken in the form $\eta=n m_{i} D$. At the inner boundary flux surface, which was located few $\mathrm{cm}$ from the separatrix, the density; the electron heat flux, ion heat flux and the average toroidal momentum flux were specified $[5,6]$. The boundary heat fluxes were imposed independently from the toroidal momentum flux thus providing the opportunity to investigate the dependence of radial electric field on these parameters $[7,9]$. The case of unbalanced counter-neutral beam injection was considered. The main results of simulation are:

1) In the first result of simulation, we illustrate the impact of neutral variation (neutral puff) on plasma parameters by solving the transport equations (Equations (4), (7) and $(29)[5,6])$ by using B2SOLPS0.5.2D fluid transport code in Figures 1-6 across the outer midplane for several

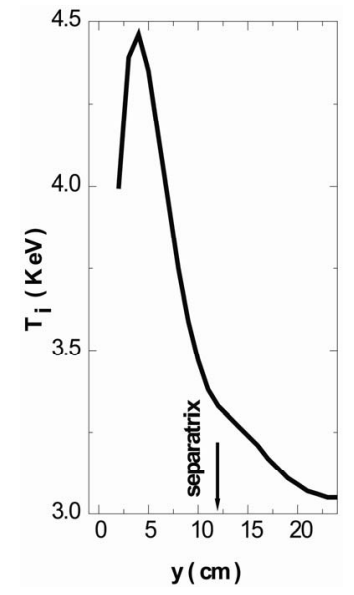

Figure 2. Ion temperature distribution in edge plasma of SSDT without gas puff.

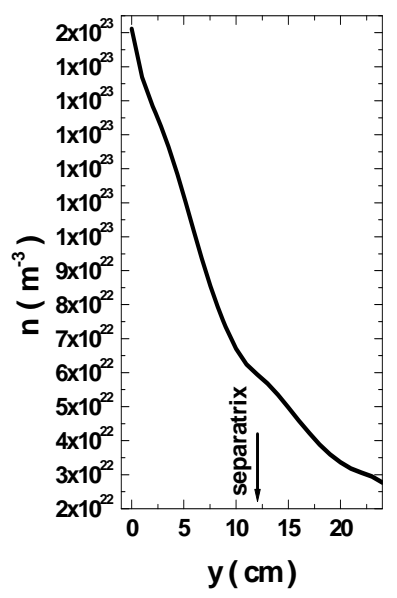

Figure 4. Plasma density distribution in edge plasma of SSDT with gas puff. 


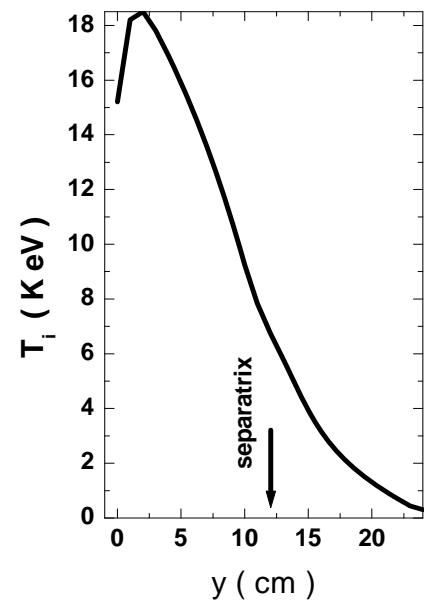

Figure 5. Ion temperature distribution in edge plasma of SSDT with gas puff.

cases with/without neutral gas puff and reverse direction of toroidal magnetic field $B_{T}$ that is $\boldsymbol{B} \times \nabla \boldsymbol{B}$ pointing outwards the $\mathrm{X}$-point. In these figures plotted are plasma parameters directly obtained from the code. These figures also show that, gas (neutral) puffing increasing the plasma density, plasma temperature and little change in toroidal (parallel) velocity in the edge plasma of small size divertor tokamak through charge exchange and ionization processes.

2) The sum of total momentum balance equation leads to [9]:

$$
\left\langle\boldsymbol{B} \cdot \nabla\left(\overleftrightarrow{\pi_{i}^{(\mathrm{Neo})}}+\overleftrightarrow{\pi_{n}}+\overleftrightarrow{\pi_{i}^{(\mathrm{AN})}}\right)\right\rangle=0
$$

where $\pi_{i}^{(\mathrm{Neo})}, \pi_{i}^{(\mathrm{AN})}$ and $\pi_{n}$ are neoclassical, anomalous and neutral viscosity tensors of ions and neutral. According to [1] the anomalous was neglected and if $n_{n} / n_{i}>$ $10^{-4}$ neutral puffing play significantly role in transport of toroidal momentum in edge plasma of this tokamak and leads to the neutral viscosity dominating over ion neoclassical viscosity. Switching on the current driven anomalous viscosity did change plasma density profile signifycantly. The second simulation result contrary to the previous result one can see the mechanism proposed in [1] doesn't play a significant role. Therefore, the anomalous transport of toroidal velocity dominates over neutral viscosity as shown in Figure 7. In the SOL far from separatrix my result consistent with the result [1]

3) According to $[5,7]$ the radial electric field is of order of neoclassical radial electric field in edge plasma of small size divertor tokamak

$$
E_{r}=E^{(N E O)}=\frac{T_{i}}{e}\left(\frac{1}{h_{y}} \frac{\mathrm{d} \ln n}{\mathrm{~d} y}+k_{T} \frac{1}{h_{y}} \frac{\mathrm{d} \ln T_{i}}{\mathrm{~d} y}\right)-b_{x} \frac{\oint \sqrt{g} V_{\|} B \mathrm{~d} x}{\oint \sqrt{g} \mathrm{~d} x}
$$

where $\sqrt{g}=h_{x} h_{y} h_{z}$ is the metric coefficients,

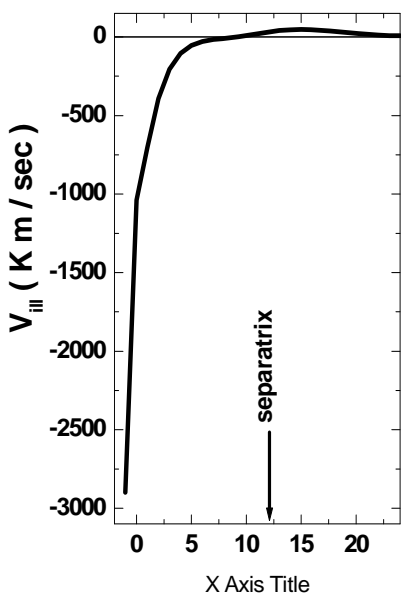

Figure 6. Parallel velocity distribution in edge plasma of SSDT with gas puff.

$\left(h_{x}=1 / \hat{e} \tilde{N} x \hat{e}, h_{y}=1 / \hat{e} \tilde{N} y \hat{e}, h_{z}=1 / \hat{e} \tilde{N} z \hat{e}\right), V_{\|}$is the parallel (toroidal), $x$ is poloidal coordinate, $y$ is radial coordinate and the coefficient $k_{T}=2.7$ corresponds to Pfirsch-Schluter regime. Equation (2) can be written in the form:

$$
E_{r}=E^{(N E O)}=\frac{T_{i}}{e} \cdot \frac{1}{h_{y}} \frac{\mathrm{d} \ln n}{\mathrm{~d} y}\left(1+k_{T} \eta_{i}\right)-b_{x} \frac{\oint \sqrt{g} V_{\|} B \mathrm{~d} x}{\oint \sqrt{g} \mathrm{~d} x}
$$

where $\eta_{i}=\frac{\mathrm{d} \ln T_{i}}{\mathrm{~d} y} / \frac{\mathrm{d} \ln n}{\mathrm{~d} y}$ here, only the temperature and density gradient terms depends on gas (neutral) puff, so that the overall sensitivity of $E_{r}$ to the gas(neutral) puff depends on the relative size of the density and temperature gradients. The third result of simulation represented in Figure 8 shows that, the factor $\eta$ is greater than unity makes the effect of gas (neutral) larger than in short mean free path. Therefore, the effect of gas (neutral) puff is large and depends on the relative size of density and temperature. The third result of simulation show that, the radial electric field of edge plasma of small size divertor tokamak has significantly affected by gas (neutral) puff as shown in Figure 8. During the gas (neutral) puffing additional radial electric field associate with ionization source is generated in edge plasma of small size divertor tokamak (Figure 8).

4) The total heat flux across the flux surfaces towards the " $y$ " (radial coordinate [5,7]) or across the surface perpendicular to the flux surface towards the " $x$ " (poloidal coordinate [5,7] in the computation region are obtain in B2-SOPLS0.5.2D modeling. The modeling fourth result show that, the gas (neutral) puff in the edge plasma of small size divertor tokamak has significantly influence on the peak values and distributions of plasma heat flux in edge plasma of this tokamak as shown in Figures 9-12. Figures 9-12 show that, the gas (neutral) puff has signifycantly effect on the distribution of plasma heat flux in edge plasma of small size divertor tokamak through re- 


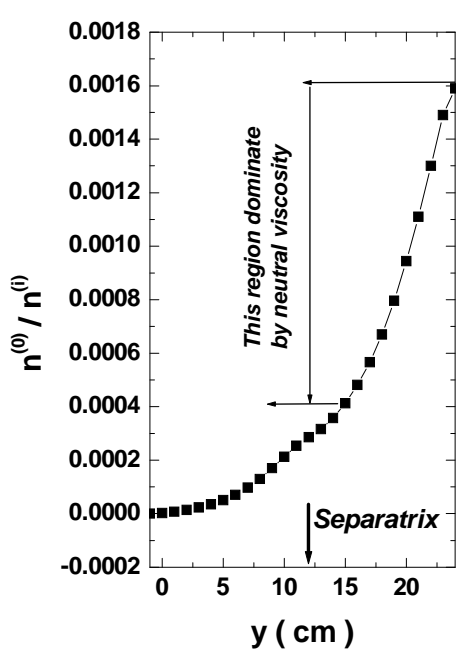

Figure 7. Distribution of ratio between density of neutral and density of ions in edge plasma of SSDT with gas puff.

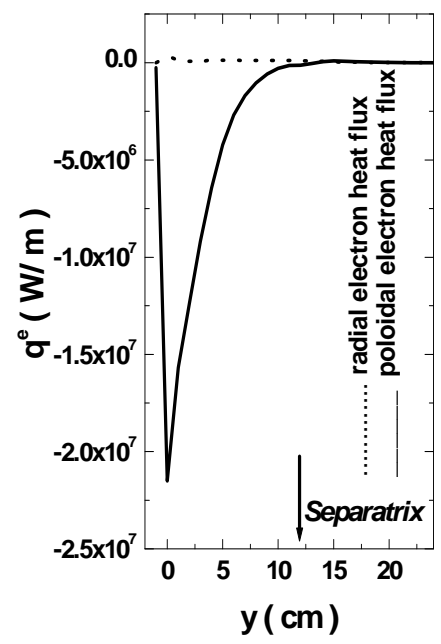

Figure 9. The distribution of poloidal and radial electron heat flux without gas puffing.

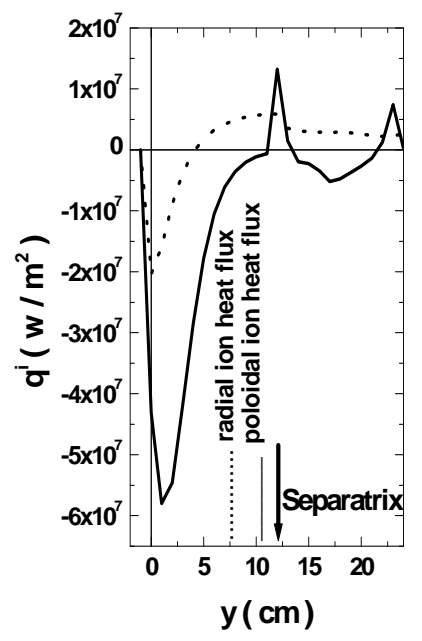

Figure 11. The distribution of poloidal and radial ion heat flux without gas puffing.

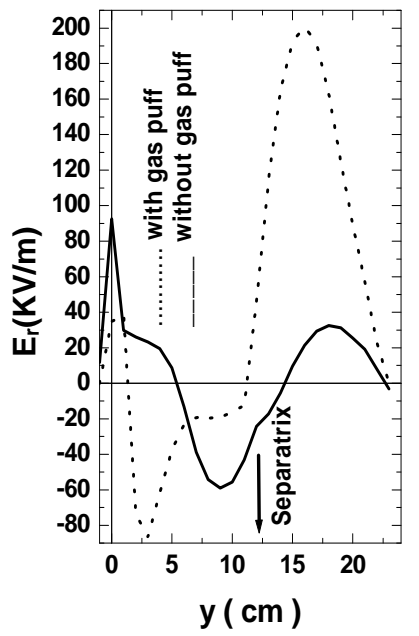

Figure 8. Radial electric field distribution in edge plasma of SSDT with and without gas puff.

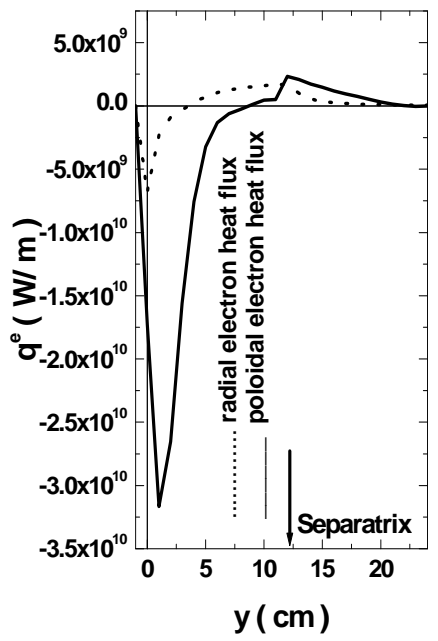

Figure 10. The distribution of poloidal and radial electron heat flux with gas puffing.

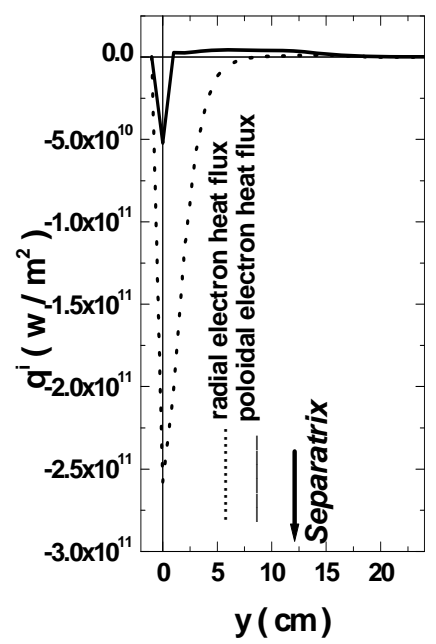

Figure 12. The distribution of poloidal and radial ion heat flux with gas puffing. 
ducetion of heat loads on the divertors plates and external wall. Large peak values of poloidal electron heat flux, which a major part of plasma heat towards to the target plate and small half width of the peak values at the outer midplan as shown in Figures 9-12.

5) The internal transport barrier (ITB) formation is key element of transition into improved confinement regimes (H-regime). Experiments on many tokamaks demonstrate that, the formation of internal transport barrier is connected with the suppression of turbulent transport by radial electric field shear. Therefore, the calculation of the radial electric field shear is very important. In this paper the radial electric field shears and internal barrier formation are the simulated by B2SOLPS0.05.2D code. It is found that, strong internal transport barrier formed and near separatrix the radial electric field shear are strong affect by neutral gas puffing as shown as in Figures 13, 14. This result is interesting since they might help explain the easier to H-regime with gas puff in SSDT. The

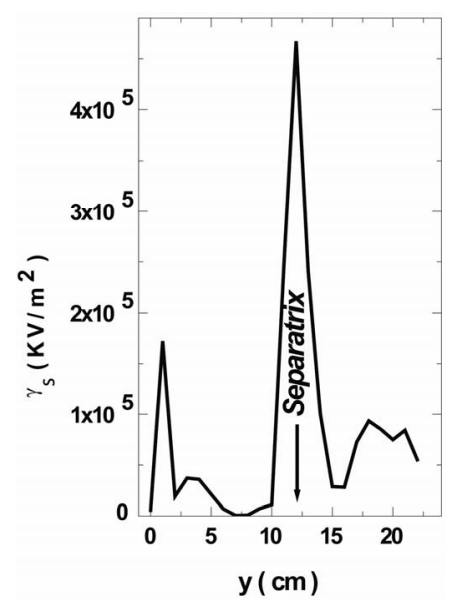

Figure 13. The radial electric field shear in edge plasma of small size divertor tokamak with gas puffing.

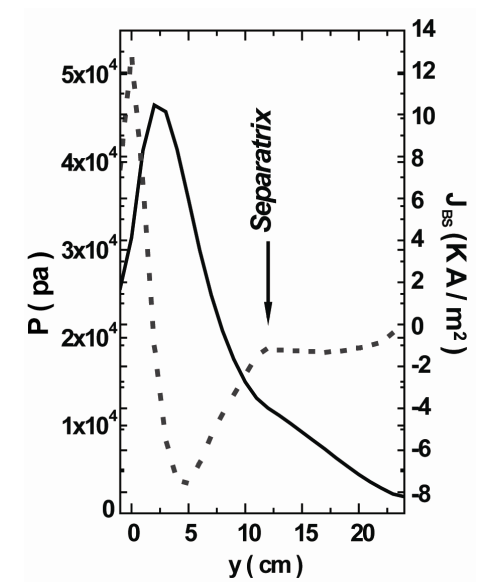

Figure 15. Radial distribution of plasma kinetic pressure and bootstrap current in the edge plasma of small size divertor tokamak without gas puffing. easier H-regime access could, for instance be caused by suppression of edge turbulence by torodial flow shear inside the separatrix where anomalous viscosity dominates.

6) Apart from the criteria for the optimization of confinement and fusion power density. Another condition regarding the operation of a tokamak in steady state is the requirement for a large fraction of bootstrap (noninductive). The maximization of the intrinsic bootstrap current is essential for very for weakens the magnetic shear and contributes to $\mathrm{L}-\mathrm{H}$ transition and stability in tokamak plasma edge [10]. Simulation result shows that, for gas (neutral) puffing the strong ITB formed at maximum bootstrap current is strong affect by neutral puffing as show as in Figures 15, 16. This result is interesting since they might show that, the strong ITB formation and plasma stability due to large bootstrap current induces in edge plasma of small size divertor tokamak by gas (neutral) puffing.

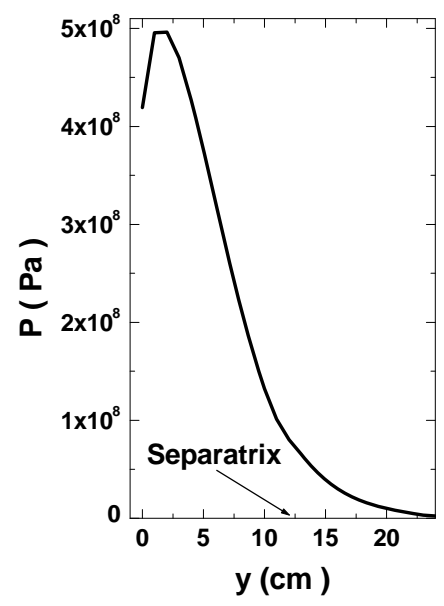

Figure 14. The plasma pressure in edge plasma of small size divertor tokamak with gas puffing.

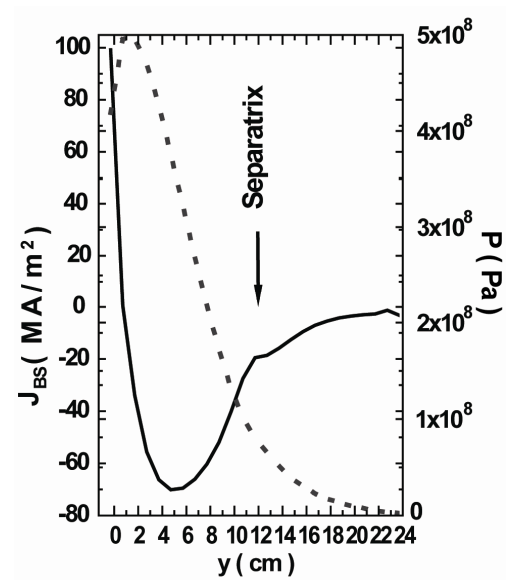

Figure 16. Radial distribution of plasma kinetic pressure and bootstrap current in the edge plasma of small size divertor tokamak with gas puffing. 


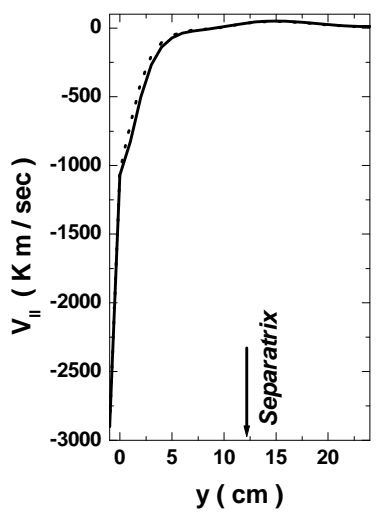

Figure 17. The radial profile of toroidal (parallel) velocity during neutral gas puffing when switching on and off centrifugal effects.

7) Switching on and off the current driven by centrifugal effect did not change the radial distribution of toroidal (parallel) velocity during neutral gas puffing as shown in Figure 17.

\section{Conclusions}

The simulation results demonstrated the following results:

1) The Plasma Parameters are significantly effect by gas (neutral) puffing in the edge plasma of small size divertor tokamak.

2) Contrary to previous work of the predominant role of neutral viscosity on toroidal flux, anomalous transport was found to be mainly effect on toroidal flux.

3) Additional radial electric field associated with ionization source generated by gas (neutral) puff in edge plasma of small size divertor tokamak.

4) Switching on and off the current driven by centrifugal effect did not change the radial distribution of toroidal (parallel) velocity during neutral gas puffing.

5) The neutrals puffing in the edge plasma of small size divertor tokamak has significantly effect on the peak values and distributions of plasma heat flux in edge plasma of this tokamak. Large peak values of poloidal electron heat flux, which a major part of plasma heat towards to the target plate and small half width of the peak values at the outer midplan. This result is interesting since they might help explain very high heat flux exists near to the separatrix needs special design for the target plate, including the choice of material, and the design of cooling system, is necessary.

6) The radial electric field shear which is the key issue for the L-H transition is strong affect by neutral gas puffing. This result is interesting since they might help explain the easier to H-regime with gas puff in SSDT. The easier H-regime access could, for instance be caused by suppression of edge turbulence by torodial flow shear inside the separatrix where anomalous viscosity dominates.
7) The bootstrap current which is the key issue for weakens the magnetic shear and contributes to $\mathrm{L}-\mathrm{H}$ transition and stability in tokamak plasma edge is signifycantly affected by neutral puffing. This result is interesting since they might help explain the strong ITB formation and plasma stability due to large bootstrap current induces in edge plasma of small size divertor tokamak by gas (neutral) puffing.

\section{REFERENCES}

[1] T. Fulop, P. J. Catto and P. Helander, "Localized Gas Puffing Control of Edge Rotation and Electric Field," Contribution to Plasma Physics, Vol. 44, No. 1-3, 2004, pp. 281-283.

[2] A. R. Field, P. G. Carolan, P. J. Catto1, N. J. Conway, G. F. Counsell and T. Fülöp, "The Influence of Neutrals on the L/H-Transition," 30th EPS Conference on Plasma And Controlled Fusion, Petersburg, 7-11 July 2003, p. 3.165 .

[3] V. Rozhansky, E. Kaveeva, S. Voskoboynikov, G. Counsell, A. Kirk, D. Costet and R. Schneider, "Generation of Toroidal Rotation by Gas Puff Simulation of Mast Experiments with B2SOLPS5.0," Journal of Nuclear Mater, Vol. 337-339, 2005, pp. 291-295. doi:10.1016/j.jnucmat.2004.10.080

[4] J. Ghosh, H. R. Griem, R. C. Elton, J. L. Terry, E. Marmar, B. Lipschultz, B. LaBombard, J. E. Rice and J. L. Weaver, "Measurements of ion And Neutral Atom Flows and Temperatures in the Inner and Outer Midplane ScrapeOff Layers of the Alcator C-Mod Tokamak," Physics of Plasma, Vol. 11, No. 3, 2004, p. 1033. doi:10.1063/1.1646394

[5] V. Rozhansky, E. Kaveeva, S. Voskoboynikov, G. Counsell, A. Kirk, D. Costet and R. Schneider, "Simulation of Tokamak Edge Plasma Including Self-Consistent Electric Field,” Nuclear Fusion, Vol. 41, No. 4, 2001, p. 387. doi:10.1088/0029-5515/41/4/305

[6] A. H. Bekheit, "Simulation of Small Size Divertor Tokamak Plasma Edge Including Self-Consistent Electric Fields,” Journal of Fusion Energy, Vol. 27, No. 4, 2008, pp. 338345. doi:10.1007/s10894-008-9148-z

[7] A. H. Bekheit, "Modelling of Small Size Divertor Tokamak Plasma Edge in the Higher Confinement Regime," Journal of Fusion Energy, Vol. 30, No. 4, 2011, pp. 352356. doi:10.1007/s10894-011-9386-3

[8] T. D. Rognlien, D. D. Ryutov and N. Mattor, "Calculation of 2-D Profiles for the Plasma and Electric Field near a Tokamak Separatrix," Czechoslovak Journal of Physics, Vol. 48, Suppl. S2, 1998, pp. 201-206.

[9] V. Rozhansky, E. Kaveeva, S. Voskoboynikov, D. Coster, $\mathrm{X}$. Bonnin and R. Schneider, "Modelling of Electric Fields in Tokamak Edge Plasma and L-H Transition," Nuclear Fusion, Vol. 42, No. 9, 2002, pp. 1110-1115. doi:10.1088/0029-5515/42/9/309

[10] R. C. Wolf, "Internal Transport Barrier in Tokamak Plasma,” Plasma Physics and Controlled Fusion, Vol. 45, 2003, pp. R1-91. doi:10.1088/0741-3335/45/1/201 